\title{
Thermo- and pH-Responsive Hydrogels Based on N-Isopropylacrylamide and Allylamine Copolymers
}

\author{
Victoria Konovalova ${ }^{1}$, Yuri Samchenko ${ }^{2}$, Ganna Pobigai ${ }^{1}$, Anatoly Burban ${ }^{1}$, Zoya Ulberg ${ }^{2}$ \\ ${ }^{1}$ Department of Chemistry, National University of Kiev-Mohyla Academy, Kiev, Ukraine \\ ${ }^{2}$ Institute of Biocolloidal Chemistry, National Academy of Sciences of Ukraine, Kiev, Ukraine \\ Email:vita@ukma.kiev.ua
}

Received October 17, 2013; revised November 19, 2013; accepted December 5, 2013

Copyright (C) 2013 Victoria Konovalova et al. This is an open access article distributed under the Creative Commons Attribution License, which permits unrestricted use, distribution, and reproduction in any medium, provided the original work is properly cited.

\begin{abstract}
The thermo- and $\mathrm{pH}$-responsive hydrogels were synthesized via copolymerization of $\mathrm{N}$-isopropylacrylamide and allylamine hydrochloride monomers. The equilibrium swelling of the hydrogels was studied as a function of temperature and $\mathrm{pH}$ in aqueous solutions. It was shown that controlled alteration of the hydrogel phase transition temperature can be achieved by changing their composition and $\mathrm{pH}$ of the environment. Increase in content of hydrophilic allylamine from 10 to $60 \mathrm{wt} \%$ in monomer mixture causes a shift of the phase transition temperature from $35^{\circ} \mathrm{C}$ to $47^{\circ} \mathrm{C}$. Hydrogels with $\mathrm{N}$-isopropylacrylamide/allylamine hydrochloride mass ratio of 3:2 show the highest $\mathrm{pH}$-response. Values of average molecular weight between polymer cross-links, $\bar{M}_{c}$, and Flory parameter, $\chi$, were calculated using temperature dependences of the equilibrium swelling of the synthesized hydrogel.
\end{abstract}

Keywords: Thermo-Responsive Hydrogels; Copolymer Hydrogels; Allylamine Hydrochloride; N-Isopropyl-Acrylamide; The Parameter of Flory

\section{Introduction}

Polymer hydrogels are one of the most promising biomedical materials in controlled drug delivery, immunoassays, biotechnology, separation processes, etc. [1-3]. Stimuli-responsive hydrogels are of special interest enabling a variety of approaches in the development of smart materials. Thermo-sensitive hydrogels undergo an abrupt structural transition from the swollen to collapsed state at transition temperature $\left(T_{p}\right)$. Important properties of thermosensitive hydrogels are temperature range, at which the network collapses, as well as the rate of the system response to the outer stimulus.

Poly-N-isopropylacrylanide (PNIPA) is one of the most studied temperature-sensitive polymers [3-7]. At room temperature, polymer chains of (co)polymers based on NIPA adopt expanded conformation with high affinity to water due to intensive hydrogen bonding. Solutions are homogenous and clear [8]. As the temperature increases, molecular vibrations weaken $\mathrm{H}$-bonds and water molecules migrate from the polymer. Hydrophobicity of the polymer chains increases and eventually this change initiates phase separation due to polymer aggregation and precipitation when temperature is higher than $T_{p}$ [7]. The phase transition is reversible and is initiated by the slight temperature changes in both directions within $T_{p}$ region. Similar transition takes place in hydrogels, which are cross-linked materials. Phase transition of poly-NIPAbased hydrogels occurs in a narrow temperature range between $32^{\circ} \mathrm{C}$ and $34^{\circ} \mathrm{C}$. This temperature interval is very close to the temperature of a human body, though not reaching it, which limits some biomedical applications of thermo-sensitive hydrogels. Other disadvantages of the poly-NIPA-based hydrogels include their poor mechaniccal strength and weak pH sensitivity.

There are several ways to influence the transition temperature by controlling the hydrophilic-hydrophobic balance of a macromolecular system. This is achievable by changes in composition of both aqueous media and polymer molecular structure. They include variations in the solution ionic strength [4], introduction of surfactants [5] or changes in ratio of hydrophilic and hydrophobic units in the hydrogel polymer structure [6,7]. These factors also influence the temperature range of the hydrogel thermo-stimulated response [9]. Both an abrupt discrete $[10,11]$ and discontinuous [12] decrease in swelling degree at temperature rise are described in literature. Thus 
it was shown that continuous phase transition transforms into discrete one with the decrease in cross-linking density of NIPA-based hydrogels [13]. Non-ionized NIPAbased gels are characterized by continuous phase transition, while phase transformation of ionized hydrogels is of discrete nature [14]. On the contrary, only continuous phase transition was observed for NIPA/acrylamide (AA) hydrogels [15]. Such a behavior was explained by the weakening of the aggregation of dehydrated NIPA chains at high temperatures due to the presence of highly hydrophilic acrylamide moieties. Several publications $[9,16]$ deal with mathematical modeling of the above-mentioned process, though this problem is still unclear and needs father investigation.

Thermosensitivity can be combined with $\mathrm{pH}$-sensitivity via introduction of ionic moieties into the hydrogel structure $[17,18]$. Such a combination enhances purposeful controlling of hydrogels properties and thereby their applications. Incorporation of residues containing weak acidic groups causes the hydrogel collapse at lower $\mathrm{pH}$ and, vice versa, weak basic groups in the polymer structure lead to the phase transition at higher $\mathrm{pH}$ values. Temperature and $\mathrm{pH}$ influence on physical and chemical properties of NIPA co-polymers with non-ionogenic monomers (acrylamide, acrylonitrile, methylacrylate) and with ionogenic acidic monomer (acrylic acid) were studied in detail in $[19,20]$.

In this study, hydrogels based on NIPA copolymer with a basic monomer, allylamine, have been synthesized. Contrary to vinyl monomers allyl ones are known to polymerize with little yields due to high stability of the relevant radical and prevailing side reactions. In the present work, corresponding copolymer hydrogels were synthesized using allylamine hydrochloride.

Introduction of AlAm component improves the mechanical strength of PNIPA hydrogels and adds $\mathrm{pH}$-sensivity to the system. The effect of external stimuli such as the $\mathrm{pH}$ and temperature on the equilibrium swelling ratios of these hydrogels was investigated. Values of average molecular weight between polymer cross-links, $\bar{M}_{c}$, and Flory parameter, $\chi$, were calculated using temperature dependences of the equilibrium swelling of the hydrogel synthesized. Such hydrogels have potential in the development of smart both $\mathrm{pH}$-and thermo-responsive membranes, systems for targeted drug delivery, sensors, etc.

\section{Experimental}

Sigma-Aldrich reagents were used to synthesize the hydrogels. NIPA was recrystallizated from hexane. A crosslinking agent, N,N-methylenebisacrylamide (MBA), and components of redox initiating system, ammonium persulphate (APS) and tetramethylethylenediamine (TEMED), were used without further purification. Allylaminehydrocloride $(\mathrm{AlAmH})$ was synthesized via allylamine reaction with gaseous hydrochloride acid. $30 \mathrm{~g}$ of allylimine were dissolved in $300 \mathrm{~cm}^{3}$ of anhydrous diethyl ether and cooled to $-15^{\circ} \mathrm{C}$. Gaseous $\mathrm{HCl}$ was allowed to bubble through the solution for one hour until white precipitate of allylamine hydrochloride was formed.

White AlAmH crystals were filtered out and dried under vacuum at room temperature. Molecular structure of the product obtained was confirmed NMR spectroscopy. NMR spectra of allylamine hydrochloride are given in Figure 1 Contrary to unprotonated monomer (see spectral base of given organic compounds SDBS, No. $4258 \mathrm{H}$ SP-00-733)[http://riodb01.ibase.aist.go.jp/sdbs/cgi-bin/di rect_frame_top.cgi], amino-group protons of which posses singlet with chemical shift 1.29 m.d., corresponding allylamine hydrochloride protons are characterized by singlet at 8.4 m.d.(Figure 1).

\subsection{Synthesis of P(NIPA-co-AIAm) Hydrogels}

P(NIPA-co-AlAmH) hydrogels were synthesized by freeradical crosslinking copolymerization of NIPA and AlAmH in aqueous solutions. APS $(0.056 \mathrm{M})$ and TEMED $(0.32$ M) were used as the redox initiator system (see Scheme 1).

Cross-linking agent content was $0.125 \mathrm{wt} \%$ with respect to monomer amount and a total monomer concentration was $21 \mathrm{wt} \%$. AlAmH concentration in the mixture with NIPA was varied in the range between 0 to $60 \mathrm{wt} \%$. Monomer concentrations and the corresponding NIPA/ AlAm mass ratio of synthesized hydrogels are summarized in Table 1. Polymerisation was performed at about $0^{\circ} \mathrm{C}$ in argon atmosphere.

\subsection{Characterization}

\section{NMR}

NMR spectra were obtained using the spectrometer Bruker 400 Avance (1 H $400.08 \mathrm{MHz}$ ) with dimethylsulfoxide as a solvent. Impulse duration constituted 12.15 microseconds, the receiver amplification being 39, accumulation time $-2.25 \mathrm{sec}$, and measurement temperature $-293 \mathrm{~K}$.

\section{Swelling Measurements}

For the swelling measurements, the hydrogels were synthesized, washed in distillated water and then neutralized by $2 \mathrm{M} \mathrm{NaOH}$ during $24 \mathrm{~h}$ and then washed again up to $\mathrm{pH}=7$. Netralized hydrogels were dried and then immersed in buffer solution at $\mathrm{pH}$ at 1.69, 6.86 and 9.18 that corresponding ionic strength $0.05 ; 0.1$; and 0.03 $\mathrm{mol} / \mathrm{dm}^{3}$ respectively. Equilibrium swelling ratio was determined in the temperature range from $5^{\circ} \mathrm{C}$ to $53^{\circ} \mathrm{C}$. The hydrogels were weighed at different times until the hydrated weight remained constant. Swelling ratio was 


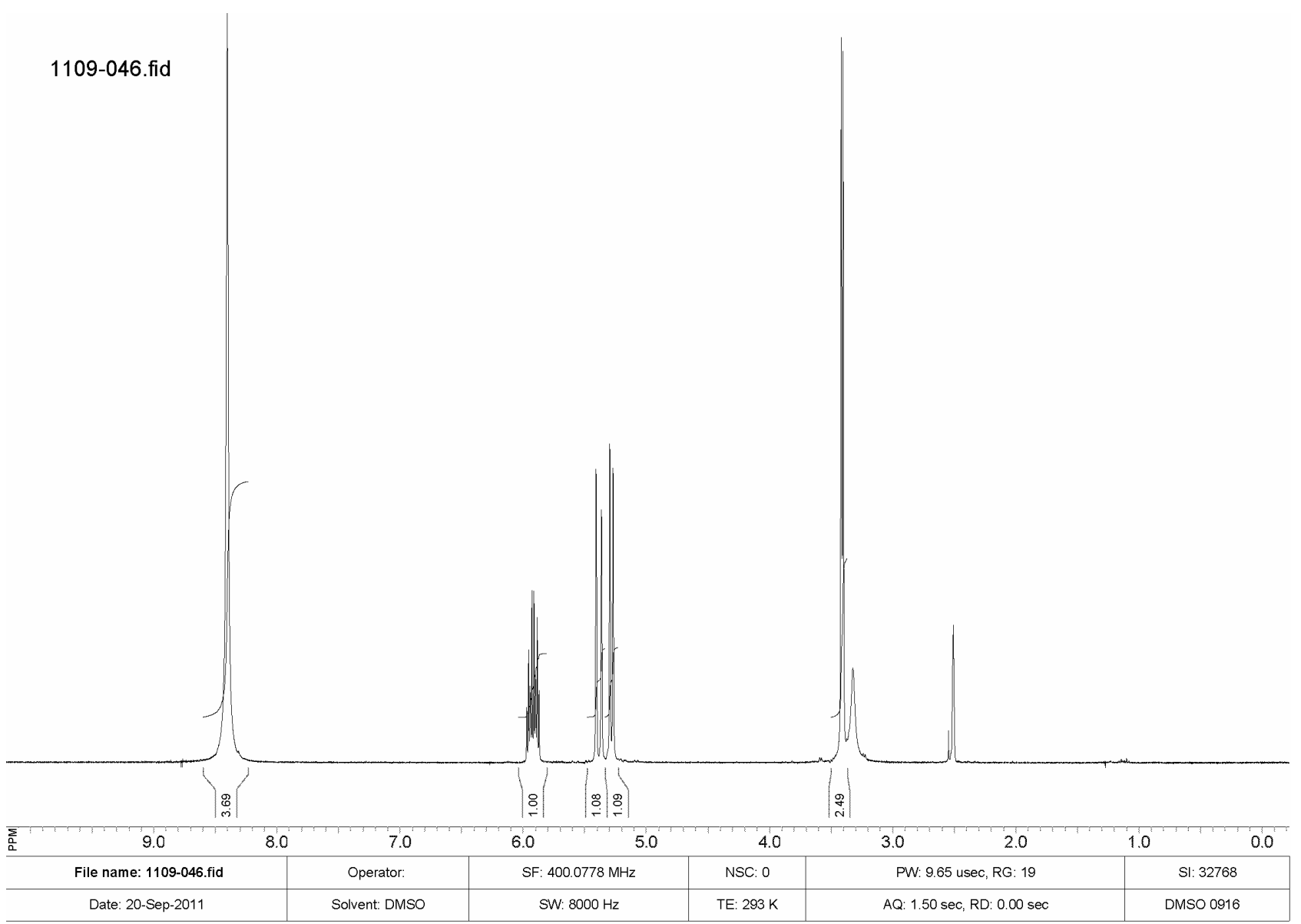

Figure 1. NMR spectra of allylamine hydrochloride.
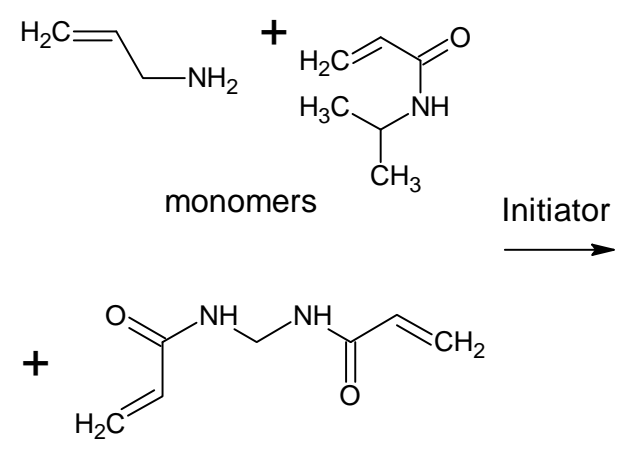

crosslinking agent

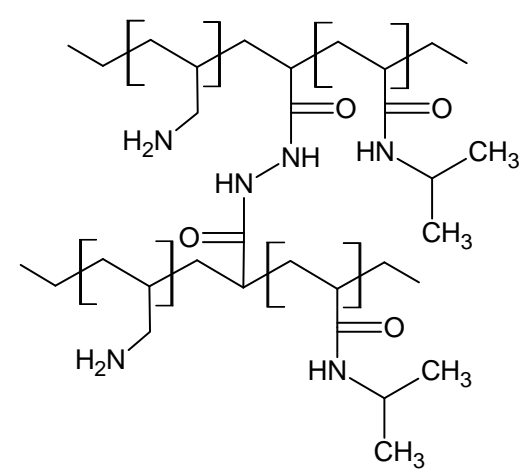

Scheme 1. P(NIPA-co-AIAm) hydrogels formation.

Table 1. Hydrogel component content (wt\%) in feed.

\begin{tabular}{cccc}
\hline Hydrogel name & NIPA & AlAmH & MBA \\
\hline P(NiPA) & 21.05 & - & 0.125 \\
P(NIPA-co-AlAm) 9:1 & 18.95 & 2.10 & 0.125 \\
P(NIPA-co-AlAm) 4:2 & 16.84 & 4.21 & 0.125 \\
P(NIPA-co-AlAm) 3:2 & 12.63 & 8.42 & 0.125 \\
P(NIPA-co-AlAm) 2:3 & 8.42 & 12.63 & 0.125 \\
\hline
\end{tabular}

determined as an average value of 10 parallel measurements calculated from the following formula:

$Q=\left(m_{s}-m_{d}\right) / m_{d}$, where $m_{s}$ is the mass of hydrogel swollen to the equilibrium state and $m_{d}$ is mass of the corresponding dried copolymer.

Thermogravimetric/Differential Thermalanalysis (TG/DTA)

The dynamic weight loss tests were conducted on 
thermogravimetric analyzer (TGA) Derivatograph Q$1500 \mathrm{D}$ for $50 \mathrm{mg}$ samples with heating rate $10^{\circ} \mathrm{C} / \mathrm{min}$ in the $20^{\circ} \mathrm{C}-300^{\circ} \mathrm{C}$ temperature range. Measurements were performed under nitrogen atmosphere with the simultaneous removal of evolved products. Temperature intervals of weight losses were estimated from the differential curves taking into account the fact that distinguishing the stages on DTA curves are more efficient (overrate capabilities) than the integral curves of the weight loss (TG). Moreover the area under DTA curve is proportional to the weight loss at a corresponding stage.

\section{Results and Discussion}

TGA analysis was conducted in the temperature range of $20^{\circ} \mathrm{C}$ to $300^{\circ} \mathrm{C}$. As it can be seen from the DTG curves (Figure 2), the obtained hydrogels have one basic stage of the weight loss ranged from $50^{\circ} \mathrm{C}$ to $110^{\circ} \mathrm{C}$ with the maximum rate of weight loss at approximately $80^{\circ} \mathrm{C}$.

This corresponds to the evaporation of weakly associated water. The quantity of the weakly associated water depends on the composition of hydrogels. NIPA hydrogel without ionic monomer is characterized by the weight loss of about $64 \%$. On incorporating $10 \mathrm{wt} \%$ and $40 \mathrm{wt} \%$ of $\mathrm{AlAmH}$ into hydrogel structure (hydrogel composition P(NIPA-co-AlAm) 9:1 and 2:3, respectively), quantity of the weakly associated water sharply increases up to $78 \%$ and $85 \%$, respectively due to the incorporation of highly hydrophilic domains containing amino-groups. Further increase of AlAmH content up to $60 \%$ (hydrogel $\mathrm{P}(\mathrm{NIPA}-\mathrm{co}-\mathrm{AlAm}$ ) 2:3) results in decreasing content of weakly associated water to about $68 \%$. Despite the considerable rise of weakly associated water quantity in the hydrogels, copolymers containing allylamine are characterized by much better elastic and mechanical properties as compared to NIPA homopolymers.

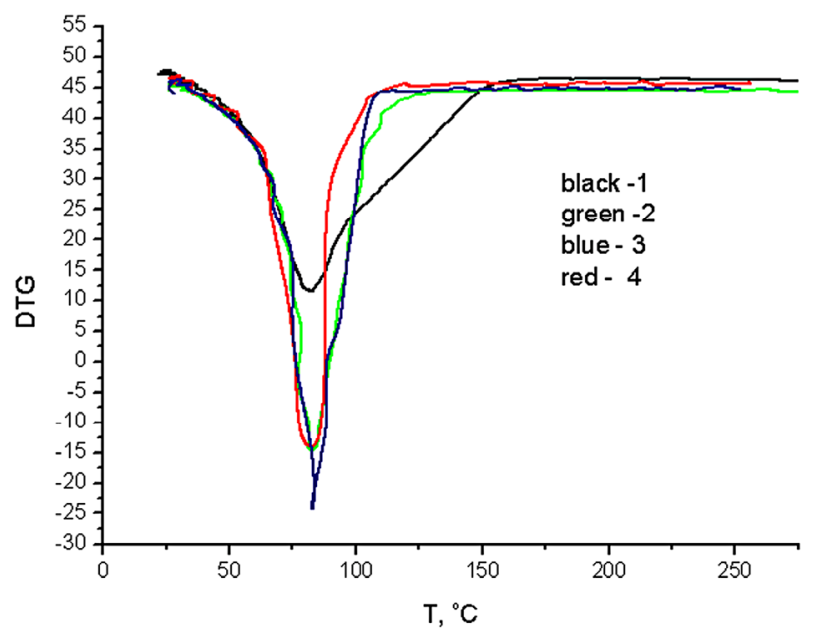

Figure 2. DTG curve of various hydrogels: $1-\mathrm{P}(\mathrm{NiPA})$; 2-P(NIPA-co-AlAm) 9:1; 3-P(NIPA-co-AlAm) 3:2, 4P(NIPA-co-AIAm) 2:3.
Figure 3 shows that synthesized PNIPA hydrogels reveal predicted sharp phase transition in the temperature range between $25^{\circ} \mathrm{C}$ to $32^{\circ} \mathrm{C}$. This coincides with the literature data [4]. The highest swelling degree reaches up to $16 \mathrm{~g}$ of a liquid per $1 \mathrm{~g}$ of the polymer, while at temperature above $T_{p}$ swelling ratio drops to as low as 2 $\mathrm{g} / \mathrm{g}$. NIPA copolymerization with monomers of various natures allows purposeful change of their phase transition temperature and sufficiently widens the area of their applications. Thus, addition of hydrophilic domains leads to the increase of $T_{p}$, while that of hydrophobic ones causes the decrease of the relevant parameter [10]. Allylamine is a monomer containing hydrophilic amino-groups. The phase transition temperature of the hydrogels was determined as a maximum on differential curves of hydrogel swelling $\pm 1-2$ grad. Thus, incorporating $10 \mathrm{wt} \%$ of AlAmH into the hydrogel results in shifting the temperature range of the phase transition to $35^{\circ} \mathrm{C}-37^{\circ} \mathrm{C}$. Further increase in AlAmH concentration up to $20 \%$ and $40 \%$ shifts temperature range of the phase transition to $40^{\circ} \mathrm{C}-41^{\circ} \mathrm{C}$ and $47^{\circ} \mathrm{C}-49^{\circ} \mathrm{C}$, respectively. Swelling ratios of such copolymer hydrogels are also considerably higher than those of NIPA-based hydrogels. The highest swelling degree $(35 \mathrm{~g} / \mathrm{g})$ is reached for the hydrogels with P(NIPA-co-AlAm)-3:2 composition. Differential curves of hydrogel swelling (Figure 3(b)) demonstrate enhanced intensity and discreteness of the phase transition on substituting NIPA chains by allylamine up to the ratio of $3: 2$. The further increase of allylamine (P(NIPA-co-ALAm) gives continuous phase transition. Thus P(NIPA-coAlAm)-3:2 composition shows continuous phase transition. This fact is explained by the decrease of the hydrophobic aggregation of dehydrated NIPA chains at high temperatures when they appear separated by hydrophilic allylamine chains. Therefore, any further increase of allylamine is not expedient since it leads to the decrease of swelling degree and the loss of hydrogel thermo-responsive properties.

Swelling ratio is an important parameter that defines solvent quantity in the hydrogel in the equilibrium state and is the function of the polymer network structure, cross-linking degree, hydrophilysation and functional groups dissociation degree. The latter is dependent on temperature, ionic strength and $\mathrm{pH}$ of the environment. Therefore swelling ability of P(NIPA-co-AlAmH) hydrogels was studied at $\mathrm{pH} 1.68 ; 6.86$ and 9.18. Non-ionogenic PNIPA hydrogel only slightly varies the swelling degree depending on the media $\mathrm{pH}$. Also low $\mathrm{pH}$ sensitivity was observed for P(NIPA-co-AlAm) hydrogels 9:1 and 4:2. However, as Figure 4 shows, swelling of the P(NIPA-co-AlAm) hydrogel 3:2 depends on both temperature and $\mathrm{pH}$ of the environment. The highest swelling was achieved in the acidic range of $\mathrm{pH}$. At such conditions, polymer chains are in the most extended confor- 


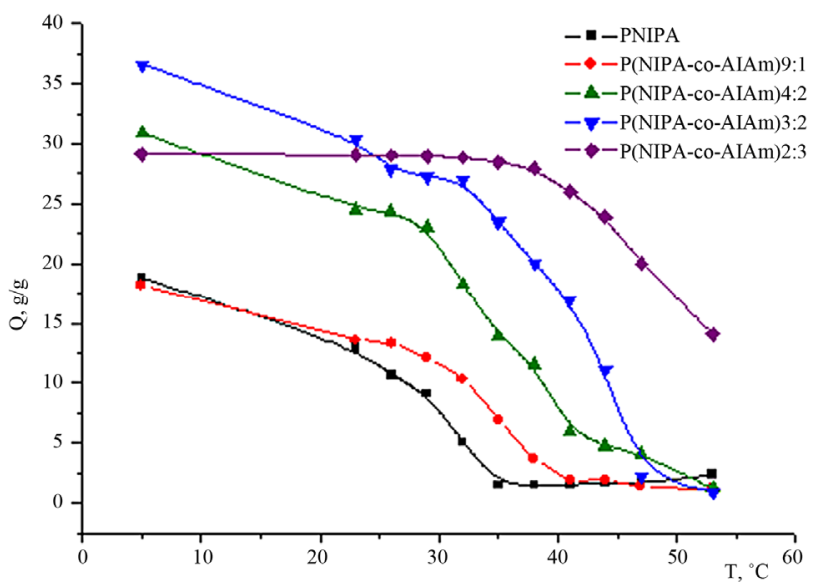

(a)

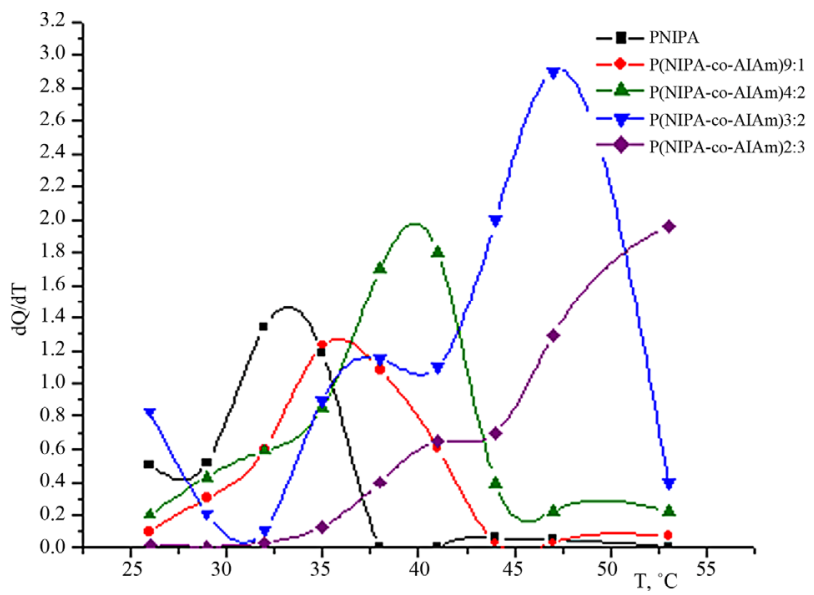

(b)

Figure 3. Integral (a) and differential (b) temperature dependences of $\mathrm{P}$ (NIPA-co-AlAm) hydrogels swelling ratio in the temperature range from $5^{\circ} \mathrm{C}$ to $55^{\circ} \mathrm{C}$ at $\mathrm{pH} 6.86$.

mation due to electrostatic repulsion between ionized $\mathrm{NH}_{3}^{+}$groups and the best conditions for macromolecular solvation are achieved.

Alkaline $\mathrm{pH}$ narrows the temperature range of phase transition and hydrogel swelling is independent of temperature above approximately $43^{\circ} \mathrm{C}$. This can be explained by the system stabilization due to inter-chain $\mathrm{H}$-bonds and their transition into more compact conformation. At low temperatures (up to $25^{\circ} \mathrm{C}$ ) the copolymer swelling is slightly dependent on $\mathrm{pH}$. The hydrogels demonstrate higher $\mathrm{pH}$-sensitivity at temperatures higher than $40^{\circ} \mathrm{C}$. Moreover, differential curves of the swelling temperature dependence on $\mathrm{pH}$ (Figure 3(b)) show that $\mathrm{pH}$ also shifts the hydrogel phase transition temperature. In the range of neutral $\mathrm{pH}$, the copolymers are characterized by the biggest change of the swelling degree and collapse temperature $47^{\circ} \mathrm{C}-49^{\circ} \mathrm{C}$. The shift of $\mathrm{pH}$ to both alkaline and acidic values leads to lowering of the phase transition temperature (to $T_{p}=41^{\circ} \mathrm{C}-42^{\circ} \mathrm{C}$ at $\mathrm{pH}-1.68$ and $T_{p}$ $=38^{\circ} \mathrm{C}-39^{\circ} \mathrm{C}$ at $\mathrm{pH}=9.18$ ).

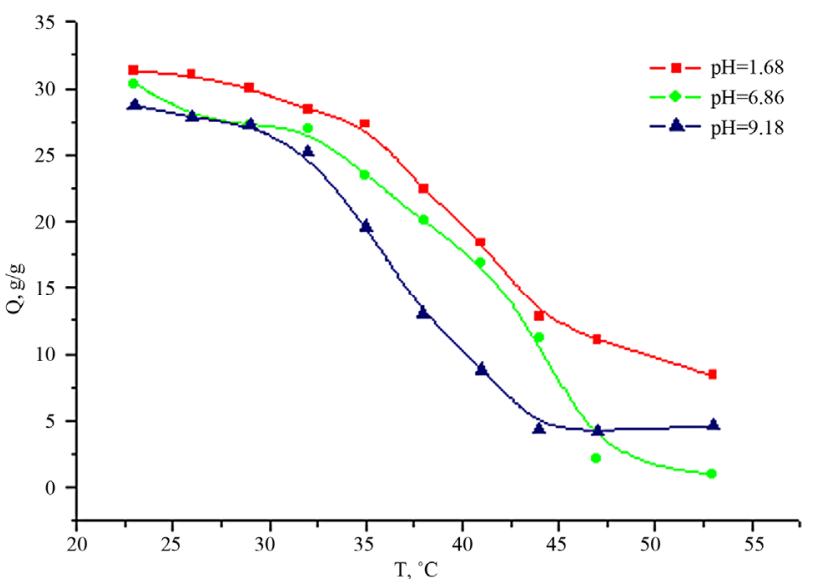

(a)

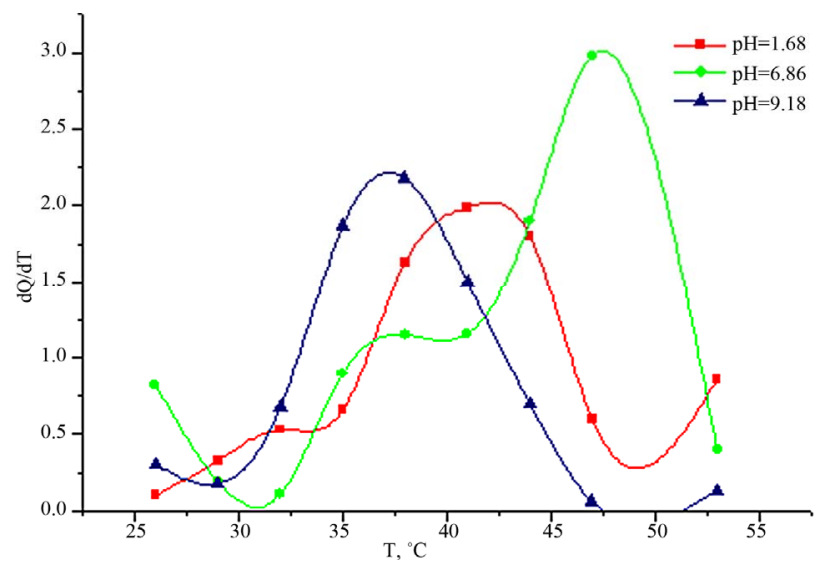

(b)

Figure 4. Integral (a) and differential (b) temperature dependences of P(NIPA-co-AlAm) hydrogel (3:2) swelling ratio in the temperature range from $5^{\circ} \mathrm{C}$ to $55^{\circ} \mathrm{C}$ at different $\mathrm{pH}$ values.

As for P(NIPA-co-AlAm) 2:3 composition, it's also $\mathrm{pH}$-responsive in phase transition temperature diapason, but rate of response on temperature changes is rather lower especially in acidic environment were the swelling of hydrogels are the most higher.

The hydrogel swelling degree was studied at various temperatures and $\mathrm{pH}$ values in order to find such parameters of macromolecular matrix as the average molecular weight between crosslinks $\bar{M}_{c}$, as well as the parameter of Flory $\chi$ that describes polymer-solvent interaction.

The Flory-Rehner [21] models describe the $M_{c}$ values for neutral polymer networks, although hydrogels may be neutral or ionic in nature. Brannon-Peppas and Peppas derived an equation to describe this ionic contribution term for both anionic and cationic hydrogels [22].

The next algorithm was used for calculating $\bar{M}_{c}$ and $\chi$ According to Flory-Rener swelling theory the osmotic pressure of hydrogel is the sum of the pressures attributed to (1) polymer-solvent mixing (mix), (2) deforma- 
tion of network chains to a more elongated state (el), and (3) the non-uniform distribution of mobile counter ions between the hydrogel and the external solution (ion) [21]:

$$
\Delta \mu=\Delta \mu_{m i x}+\Delta \mu_{e l}+\Delta \mu_{i o n} .
$$

The mixing term is satisfactorily represented by a Flory-Huggins-type expression of the form

$$
\Delta \mu_{m i x}=R T\left[\ln \left(1-v_{2 m}\right)+v_{2 m}+x v_{2 m}^{2}\right]
$$

where $R$ is a gas constant, $T$-temperature, $v_{2 m}$-volume polymer fraction in the hydrogel defined as:

$$
\begin{gathered}
V_{2 m}=\left[1+\frac{\left(q_{w}-1\right) p_{\text {polymer }}}{p_{\text {solvent }}}\right]^{-1} \\
q_{w}=\frac{\text { mass of swollen hydrogel }}{\text { mass of dry hydrogel }}
\end{gathered}
$$

and $\rho$ is polymer and solvent density.

Osmotic pressure produced due to the deformation of network chains to more elongated state:

$$
\Delta \mu_{e l}=R T\left[\frac{\left(1-\frac{2}{\Phi}\right) V_{1} p v_{2 r}^{2 / 3} v_{2 m}^{1 / 3}}{M_{c}}\right]
$$

where $\Phi=4$ - number of cross-linking chains,

$V_{1}$ is solvent molar volume, constituting $18 \mathrm{~cm}^{3} / \mathrm{mol}$ for water,

$V_{2 r}$ is polymer volume fraction in the hydrogel upon its obtaining. The last is found as follows:

$$
V_{2 r}=\left[1+\frac{(p f-1) p_{\text {polymer }}}{p_{\text {solvent }}}\right]^{-1}
$$

Osmotic pressure caused by the non-uniform distribution of mobile counter ions between the hydrogel and the external solution is calculated as:

$$
\Delta \mu_{i o n}=-V_{1} R T\left(\frac{i^{2} v_{2 m}^{2} f_{c}^{2}}{4 I V_{r}^{2}}\right)
$$

where $I$ is solution ionic strength, that equals $0.05 ; 0.1$; and $0.03 \mathrm{~mol} / \mathrm{dm}^{3}$ for $\mathrm{pH} 1.68 ; 6.86$ and 9.18 respectively.

$V_{r}$ is molecular volume of the polymer was found as follows:

$$
V_{r}=\frac{M_{N i P A}\left(1-f_{c}\right)+M_{A A} \cdot f_{c}}{\rho_{\text {polymer }}}
$$

where $f_{c}$ is mole fraction of ionic groups in allylamine.

Ionization degree was calculated by $i=\frac{K_{a}}{10^{-p H}+K_{a}}$ with $K_{a}=2 \times 10^{-10}$ for allylamine.
When hydrogel reaches swelling state balanced with a pure solvent, the solvent activity in the hydrogel become equal to the solvent activity in the pure solvent (i.e. equals 1). Thus, chemical potential of the solvent in the hydrogel becomes equal to zero. Using Equations (1)-(6) the equilibrium equation can be written as:

$$
\begin{aligned}
& {\left[\frac{K_{a}}{10^{-p H}+K_{a}}\right]^{2} \frac{V_{1} f_{c}^{2}}{4 I V_{r}^{2}}-v_{2 m}^{-2} \ln \left(1-v_{2 m}\right)-v_{2 m}^{-1} } \\
= & \chi+\frac{(1-2 / \phi) V_{1} \rho_{2} V_{2 r}^{2 / 3} v_{2 m}^{-5 / 3}}{M_{c}}
\end{aligned}
$$

The above equation can be given as the dependence: $A=\chi+\frac{B}{M_{c}}$ and by plotting the dependence in A-B coordinates it is possible to find Flory parameter and the average molecular mass between the polymer crosslinks (Figure 5).

The calculations were carry out for hydrogels at tem-

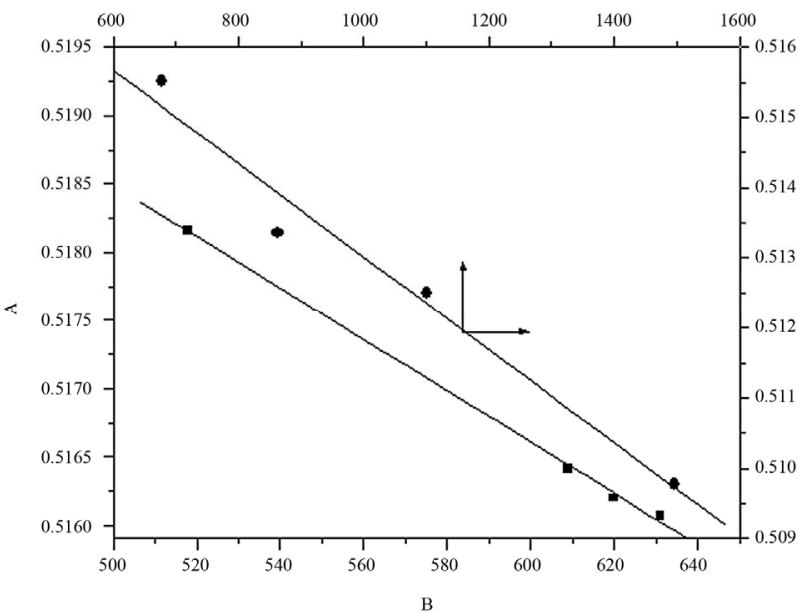

(a)

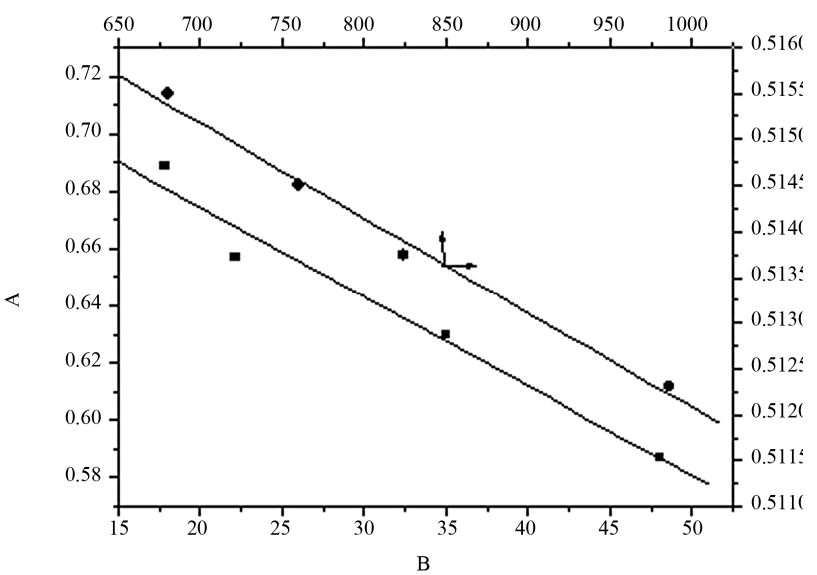

(b)

Figure 5. Determination of $\chi$ and $\bar{M}_{c}$ values of hydrogels from swelling data. (घ) P(NIPA-co-AIAm) (9:1) and (•) P(NIPA-co-AIAm) (3:2) at temperature $23^{\circ} \mathrm{C}$ (a) and $38^{\circ} \mathrm{C}$ (b). 
peratures lower and higher of $T_{p}$, such as for P(NIPA-coAlAm) (9:1) at temperature $23^{\circ} \mathrm{C}$ and $38^{\circ} \mathrm{C}$ and for hydrogel P(NIPA-co-AlAm) $(3: 2)$ at temperature $23^{\circ} \mathrm{C}, 38^{\circ} \mathrm{C}$ and $53^{\circ} \mathrm{C}$, and results were summarized in Table 2 .

The data in Table 2 show that for the P(NIPA-coAlAm) hydrogel (9:1) composition, the solvent quality change is observed at elevated temperature. It is known that the interaction parameter value $\chi$ smaller than $\sim 0.5$ indicates strong interactions between polymer macromolecules and solvent, high solvophility. On the contrary $\chi$ values exceeding 0.5 indicate materials solvophobity. Thus, in our work, P(NIPA-co-AlAm) hydrogel (9:1) with $90 \%$ content of thermo-sensitive monomer changes $\chi$ from 0.5185 to 0.6916 when temperature increases from $23^{\circ} \mathrm{C}$ to $38^{\circ} \mathrm{C}$. Moreover, $\bar{M}_{c}$ decreases by 150 times! And the $\bar{M}_{c}$ value approximates average molecular mass of copolymer chain at $38^{\circ} \mathrm{C}$. This effect can be explained by the increase of hydrophobic-hydrophobic interactions between macromolecules and additional crosslinking of the hydrogel due to $\mathrm{H}$ bonds.

With the decrease of content of hydrophobic NIPA units up to $60 \%$, solvent quality decreases insignificantly even at temperature of $53^{\circ} \mathrm{C}$. At the same time, there is 20-fold decrease in $\bar{M}_{c}$ values, which manifest as hydrogel transition to the collapsed state at the temperatures higher than $T_{p}$.

\section{Conclusion}

In the present work thermo- and pH-responsive hydrogels were synthesized via copolymerization of $\mathrm{N}$ isopropylacrylamide and allylamine hydrochloride monomers. It was shown that targeted control of the hydrogel phase transition temperature can be realized by changing their composition and $\mathrm{pH}$ of the environment. Thus rise of hydrophilic allylamine mass fraction from $10 \%$ to $60 \%$ causes shift of phase transition temperature from $35^{\circ} \mathrm{C}$ to $47^{\circ} \mathrm{C}$. Hydrogels of P(NIPA-co-AlAm) $(3: 2)$ composition are the most $\mathrm{pH}$-responsive. Values of average molecular mass between $\bar{M}_{c}$ polymer cross-links and Flory parameter $\chi$ were calculated using temperature dependences of the synthesized hydrogel swelling. It was found that the increase of hydrophilic component (AlAH) in the copolymer results in the growth of $\bar{M}_{c}$, while temperature rise causes decrease of the above-mentioned

Table 2. $\chi$ and $\bar{M}_{c}$ values for P(NIPA-co-AlAm) hydrogels.

\begin{tabular}{ccccc}
\hline Hydrogel & Temperature, ${ }^{\circ} \mathrm{C}$ & $\chi$ & $\bar{M}_{c}$ & $\mathrm{R}^{2}$ \\
\hline P(NIPA-co-AlAm) (9:1) & 23 & 0.5185 & 50000 & 0.99 \\
& 38 & 0.6916 & 323 & 0.96 \\
P(NIPA-co-AlAm) (3:2) & 23 & 0.5096 & 143000 & 0.97 \\
& 38 & 0.5117 & 100000 & 0.99 \\
& 53 & 0.5983 & 5000 & 0.87 \\
\hline
\end{tabular}

parameter. Flory parameter $\chi$ grows both with temperature and NIPA content rise in copolymer hydrogels showing intensification of hydrophilic interactions. The synthesized "smart" hydrogel systems can be used to develop novel medications, membranes, various sensors and probes that are capable of radical change of their working characteristics in response to the slightest environmental changes.

\section{REFERENCES}

[1] S. Malik, O. Boyko, N. Atkar and W. F. Young, "A Comparative Study of MR Imaging Profile of Titanium U. G. Spizzirri, I.Altimari, F. Puoci, O. I. Parisi, F Iemma, "Innovative Antioxidant Thermo-Responsive Hydrogels by Radical Grafting of Catechin on Inulin Chain," Carbohydrate Polymers, Vol. 84, No. 11, 2011, pp.517-523. http://dx.doi.org/10.1016/j.carbpol.2010.12.015

[2] I. Velthoen, J. Beek and P. Dijkstra, "Thermo-Responsive Hydrogels Based on Highly Branched Poly(Ethylene Glycol)-Poly(L-Lactide) Copolymers," Reactive and Functional Polymers, Vol. 71, No. 3, 2011, pp. 245-253. http://dx.doi.org/10.1016/j.reactfunctpolym.2010.08.007

[3] T. Caykara, "Effect of Maleic Acid Content on Network Structure and Swelling Properties of Poly(N-Isopropylacrylamide-Co-Maleic Acid) Polyelectrolyte Hydrogels," Journal of Applied Polymer Science, Vol. 92, No. 2, 2004, pp. 763-769. http://dx.doi.org/10.1002/app.20032

[4] H. Inomata, S. Goto, K. Otake and S. Saito, "Effect of Additives on Phase Transition of N-Isopropylacrylamide Gels," Langmuir, Vol. 8, No. 2, 1992, pp. 687-690. http://dx.doi.org/10.1021/la00038a064

[5] M. Meewes, J. Ricka, M. Desilva, R. Nyffenegger and T. Binkert, "Coil-Globule Transition of Poly(N-Isopropylacrylamide): A Study of Surfactant Effects by Light Scattering," Macromolecules, Vol. 24, No. 21, 1991, pp. 5811-5816. http://dx.doi.org/10.1021/ma00021a014

[6] B. Tasdelen, N. Kayaman-Apohan and M. Baysal, "Preparation, Characterization, and Drug-Release Properties of Poly(N-Isopropylacrylamide) Microspheres Having Poly(Itaconic Acid) Graft Chains," Journal of Applied Polymer Science, Vol. 97, No. 3, 2005, pp. 1115-1124. http://dx.doi.org/10.1002/app.21777

[7] G. Chen and A. S. Hoffman, "Graft Copolymers That Exhibit Temperature-Induced Phase Transition over a Wide Range of pH," Nature, Vol. 373, No. 6509, 1995, pp. 4952. http://dx.doi.org/10.1038/373049a0

[8] I. Hiroshi, G. Shuichi and S. Shozaburo, "Phase Transition of N-Substituted Acrylamide Gels," Macromolecules, Vol. 23, No. 22, 1990, pp. 4887-4888. http://dx.doi.org/10.1021/ma00224a023

[9] W. Chi and Z. Shuiqin, "Volume Phase Transition of Swollen Gels: Discontinuous or Continuous?" Macromolecules, Vol. 30, 3, 1997, pp. 574-576. http://dx.doi.org/10.1021/ma960499a

[10] S. Sasaki and S. J. Okabe, "Effects of Ions on the Solubility Transition and the Phase-Separation of N-Isopropylacrylamide in Water," Physical Chemistry B, Vol. 115, 
No. 44, 2011, pp. 12905-12910. http://dx.doi.org/10.1021/jp207576z

[11] Y. Hirose, Y. Hirokawa and T. Tanaka, "Phase Transition of Submicron Gel Beads," Macromolecules, Vol. 20, No. 6, 1987, pp. 1342-1344. http://dx.doi.org/10.1021/ma00172a029

[12] H. Yu and D. W. Grainger, "Amphiphilic Thermosensitive N-Isopropylacrylamide Terpolymer Hydrogels Prepared by Micellar Polymerization in Aqueous Media," Macromolecules, Vol. 27, No. 16, 1994, pp. 4554-4560. http://dx.doi.org/10.1021/ma00094a019

[13] Y. Li and T. J. Tanaka, "Study of the Universality Class of the Gel Network System," Journal of Chemical Physics, Vol. 90, No. 9, 1989, pp. 5161-5166. http://dx.doi.org/10.1063/1.456559

[14] S. J. Hirotsu, "Phase Transition of a Polymer Gel in Pure and Mixed Solvent Media," Journal of the Physical Society of Japan, Vol. 56, No. 1, 1987, pp. 233-242. http://dx.doi.org/10.1143/JPSJ.56.233

[15] C. Tuncer, K. Simin and D. Goekhan, "Thermosensitive Poly(N-Isopropylacrylamide-Co-Acrylamide) Hydrogels: Synthesis, Swelling and Interaction with Ionic Surfactants," European Polymer Journal, Vol. 42, No. 2, 2006, pp. 348-355.

http://dx.doi.org/10.1016/j.eurpolymj.2005.07.006

[16] L. Hua, W. Xiaogui, Li Hua, X. Wang, Z. Wang and K.Y. Lam, "Multiphysics Modeling of Volume Phase Transition of Ionic Hydrogels Responsive to Thermal Stimu- lus," Macromolecular Bioscience, Vol. 5, No. 9, 2005, pp. 904-914. http://dx.doi.org/10.1002/mabi.200500082

[17] H. Bishta, L. Wanb, G. Maob and D. Oupicky, "pHControlled Association of PEG-Containing Terpolymers of N-Isopropylacrylamide and 1-Vinylimidazole," Polymer, Vol. 46, No. 19, 2005, pp. 7945-7952. http://dx.doi.org/10.1016/j.polymer.2005.06.080

[18] H. Dautzenberg, Y. B. Gao and M. Hahn, "Formation, Structure, and Temperature Behavior of Polyelectrolyte Complexes between Ionically Modified Thermosensitive Polymers," Langmuir, Vol. 16, No. 23, 2000, pp. 9070 9081. http://dx.doi.org/10.1021/la000368+

[19] V. V. Konovalova, Yu. M. Samchenko, G. A. Pobigay, A. F. Burban and Z. R. Ulberg, "Hydrogel Membranes with $\mathrm{pH}$ - and Thermo-Responsive Parameters," Proceedings of the 15th International Symposium 'Ars Separatoria 2010', Torun, 4-7 July 2010, pp. 222-225.

[20] Yu. Samchenko, V. Konovalova, G. Pobigay, A. Burban and Z. Ulberg, "Thermo Sensitive Copolymers Hydrogels with Controlled Phase Transition Temperature," Reports of Ukrainian NAS, Vol. 8, 2011, pp. 123-129.

[21] P. J. Flory, "Principles of Polymer Chemistry," Cornell University Press, Ithaca, 1953.

[22] L. Brannon-Peppas and N. A. Peppas, "Equilibrium Swelling Behavior of pH-Sensitive Hydrogels," Chemical Engineering Science, Vol. 46, No. 3, 1991, pp. 715-722. http://dx.doi.org/10.1016/0009-2509(91)80177-Z 